\title{
A Calculation Method for the Sloshing Impact Pressure Imposed on the Roof of a Passive Water Storage Tank of AP1000
}

\author{
Daogang Lu, ${ }^{1,2}$ Xiaojia Zeng, ${ }^{1,2}$ Junjie Dang, ${ }^{3}$ and Yu Liu ${ }^{1,2}$ \\ ${ }^{1}$ School of Nuclear Science and Engineering, North China Electric Power University, Beijing 102206, China \\ ${ }^{2}$ Beijing Key Laboratory of Passive Safety Technology for Nuclear Energy, North China Electric Power University, Beijing 102206, China \\ ${ }^{3}$ China Nuclear Power Engineering Co., Ltd., Beijing 100840, China
}

Correspondence should be addressed to Xiaojia Zeng; 453672704@qq.com

Received 18 January 2016; Revised 6 April 2016; Accepted 19 May 2016

Academic Editor: Iztok Tiselj

Copyright (C) 2016 Daogang Lu et al. This is an open access article distributed under the Creative Commons Attribution License, which permits unrestricted use, distribution, and reproduction in any medium, provided the original work is properly cited.

\begin{abstract}
There is a large water storage tank installed at the top of containment of AP1000, which can supply the passive cooling. In the extreme condition, sloshing of the free surface in the tank may impact on the roof under long-period earthquake. For the safety assessment of structure, it is necessary to calculate the impact pressure caused by water sloshing. Since the behavior of sloshing impacted on the roof is involved into a strong nonlinear phenomenon, it is a little difficult to calculate such pressure by theoretical or numerical method currently. But it is applicable to calculate the height of sloshing in a tank without roof. In the present paper, a simplified method was proposed to calculate the impact pressure using the sloshing wave height, in which we first marked the position of the height of roof, then produced sloshing in the tank without roof and recorded the maximum wave height, and finally regarded approximately the difference between maximum wave height and roof height as the impact pressure head. We also designed an experiment to verify this method. The experimental result showed that this method overpredicted the impact pressure with a certain error of no more than $35 \%$. By the experiment, we conclude that this method is conservative and applicable for the engineering design.
\end{abstract}

\section{Introduction}

As the ultimate heat sink of AP1000 reactor, passive cooling system (PCS) is the key equipment to ensure the safety of nuclear power plant. The large water storage tank, installed at the top of the containment of AP1000, can supply plenty of water for the passive cooling. In the extreme condition, sloshing of the free surface in the tank may impact on the roof and jeopardize structural integrity under long-period earthquake. For the safety assessment of structure, it is necessary to calculate the impact pressure caused by water sloshing.

Since the behavior of sloshing impacted on the roof is involved into a strongly nonlinear phenomenon, calculations of the impact pressure with theoretical or numerical method currently are of difficulties. Ibrahim [1] focused on a 2D tank with simple geometry to solve the linear sloshing problems using analytical methods. A numerical model using finite element technique was presented by $\mathrm{Pal}$ et al. [2] to study the linear behavior of cylindrical tanks. Choun and Yun [3] used the velocity potential and the linear water wave theory to decompose the surface wave into multiple forms. There are some complex methods to solve the nonlinear sloshing problems. Li et al. [4] used an improved material point method (MPM) to predict the liquid impact force by a contact algorithm. Liquid sloshing experiments in a partially watered square tank were proposed to validate the results of simulation. Eswaran et al. [5] proposed a numerical method based on volume of fluid (VOF) techniques with arbitraryLagrangian-Eulerian (ALE) formulation to analyze baffled and unbaffled tanks with a nonlinear sloshing behavior. However, these researches are usually valid for simple cases with linear or weakly nonlinear liquid sloshing dynamics.

But it is applicable to calculate the height of sloshing in a tank without roof. Fujita et al. [6] utilized the velocity potential theory to analyze the liquid sloshing in the annular region of more intricate coaxial circular cylinders. Formulas 

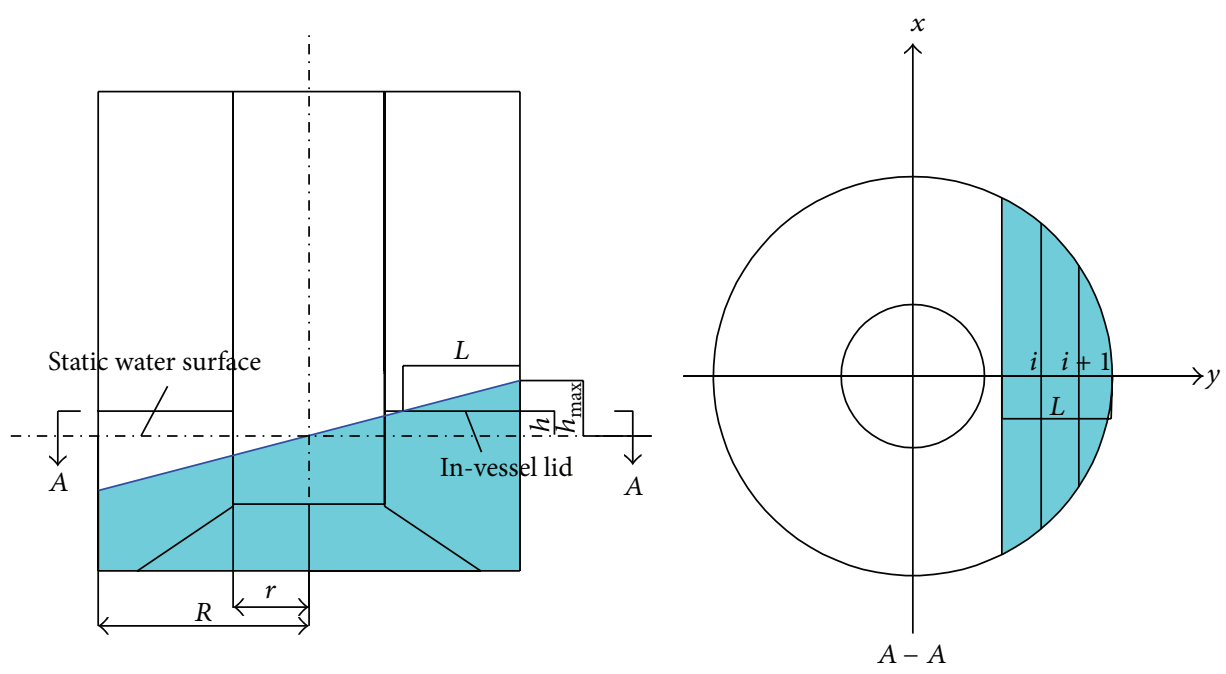

Figure 1: Schematic of sloshing.

about the maximum wave height $\left(\eta_{\max }\right)$ at shell wall and the maximum pressure $\left(p_{\max }\right)$ at the free surface were obtained. More interestingly, the correlation between maximum wave height and maximum pressure was $p_{\max }=\rho g \eta_{\max }$ from equation (34) in their research. Virella et al. [7] used the finite element package ABAQUS to investigate the free surface wave amplitude and pressure distribution of tank wall by both linear wave theory and nonlinear wave theory models. Nayak and Biswal [8] used the Galerkin-weighted-residual based finite element method (FEM) to solve Laplace equation with nonlinear boundary conditions. The wave height of nonlinear sloshing was verified to be accurate.

Besides, the impact pressure is an important parameter in the assessment of safety of engineering design. Researchers had conducted large-scale experiments to investigate impact pressure [9-12].

As is mentioned above, the available studies mostly focused on the sloshing characteristics of rectangular tanks with simple geometry. However, considering the special structure of PCCWST, which is a coaxial circular cylinder tank with an inclined bottom, it is difficult in obtaining analytical expressions for the prediction of the natural modes and the liquid motion. Moreover, numerical and analytical methods to precisely describe the sloshing impact pressure are complicated because of the significant nonlinearity phenomena. In the present paper, a simplified method was proposed to calculate the impact pressure using the sloshing wave height. Moreover, an experiment was designed to verify this method.

\section{Calculation Method}

Sloshing of the water surface in the tank may impact on the roof under long-period earthquake and the prediction of the impact pressure is necessary. Due to the apparent nonlinear behavior caused by complicated sloshing phenomena, a simplified method was proposed to calculate the impact pressure using the sloshing wave height, in which we first marked the position of the height of roof, the solid wide line showed in Figure 1, then produced sloshing in the tank without roof and recorded the maximum wave height which can be shown as the oblique line in Figure 1, and finally regarded approximately the difference between maximum wave height and roof height as the impact pressure head. In this way, the impact pressure can be calculated by the following equation:

$$
P=\rho g\left(h_{\max }-h\right)
$$

where $P$ is the maximum impact pressure, $\rho$ is the density of water, $g$ is the acceleration due to gravity, $h_{\max }$ is the maximum wave height, and $h$ is the distance between the static water surface and the roof.

Besides, the impact force of water in a tank at any time is given by the following equation:

$$
F_{w}=\iint_{s_{1}}^{s_{2}} P d s,
$$

where $F_{w}$ is impact force, $P$ is the impact pressure, and $s_{1}$, $s_{2}$, respectively, mean the lower and upper boundary of tank roof.

By utilizing the maximum sloshing wave height, the maximum impact pressure, which appeared near the outer wall, can be calculated by (1).

The impact area is divided into $n$ parts and the impact pressures in each part can be obtained by linear interpolation. The $i$ th pressure is given by the following equation:

$$
p_{i}=\frac{i}{n} \rho g\left(h_{\max }-h\right)
$$

According to Figure 1, $L$, which is related to the impact area, can be calculated by

$$
\frac{L}{R}=\frac{h_{\max }-h}{h_{\max }} .
$$


TABLE 1: Scaling factors for sloshing experiments.

\begin{tabular}{lc}
\hline Parameter & Scaling ratio \\
\hline Length: $L[\mathrm{~m}]$ & $C_{l}$ \\
Gravity: $g\left[\mathrm{~m} / \mathrm{s}^{2}\right]$ & 1 \\
Velocity: $V[\mathrm{~m} / \mathrm{s}]$ & $C_{l}^{1 / 2}$ \\
Frequency: $f[\mathrm{~Hz}]$ & $C_{l}^{-1 / 2}$ \\
Time: $t[\mathrm{~s}]$ & $C_{l}^{1 / 2}$ \\
Kinematic viscosity: $\nu\left[\mathrm{m}^{2} / \mathrm{s}\right]$ & $C_{l}^{3 / 2}$ \\
Pressure: $P[\mathrm{~Pa}]$ & $C_{\rho} C_{l}$ \\
Wave height: $H[\mathrm{~m}]$ & $C_{l}$ \\
\hline
\end{tabular}

The total impact force can be written as

$$
\begin{array}{r}
F_{t}=\sum_{i=0}^{n-1} \int_{-\sqrt{R^{2}-y^{2}}}^{\sqrt{R^{2}-y^{2}}} d x \int_{R-L+i(L / n)}^{R-L+(i+1)(L / n)} \frac{p_{i}+p_{i+1}}{2} d y \\
i=0,1,2,3, \ldots, n-1 .
\end{array}
$$

Substituting (3) and (4) to (5), we can get the total impact force.

\section{Experiment}

In order to verify the calculation method, an experiment was designed.

3.1. Scaling Model. Since the passive storage tank of AP1000 is large, experiment with the large size of tank is difficult. In order to keep the similar properties of fluid, a scaling model is proposed. For the case of the present experiment, some main governing dimensionless numbers that should be respected are as follows $[6,13]$.

Froude number: $\mathrm{Fr}=V^{2} / g L$; Reynolds number: $\mathrm{Re}=$ $V L / v$; Strouhal number: St $=f L / V$; Euler number: $\mathrm{Eu}=$ $P / \rho V^{2}$; Bond number: Bo $=\rho g L^{2} / \sigma$.

With the analysis, the Froude number, Strouhal number, Euler number, and Bond number are respected and the Re number is relaxed when ignoring viscosity effects. The scaling factors for the different parameters in sloshing experiments are summarized in Table 1 and these expressions are called the modified linear scaling method.

3.2. Experimental Equipment. According to the similarity criteria, an experimental model (Figure 2) of PCCWST was built with the scale of $1: 40$. The experimental tank was mainly cylindrical ( $0.122 \mathrm{~m}$ internal radius) with an inclined bottom and the vessel was made of acrylic sheet to observe the sloshing phenomena clearly. The tank was considered to be rigid due to the $15 \mathrm{~mm}$ thickness of outer wall. This assumption was made to simplify the study of sloshing. In order to measure the maximum sloshing wave height, the length scaling for the height of the tank was $3: 40$. A summary of the main sizes of experimental tank is presented in Figure 3.

The internal structures, showed as the red parts in Figure 3, were added suspended from the top cover, which was fixed on the outer structure. Such internal structures contained a supporting configuration and an in-vessel circle roof

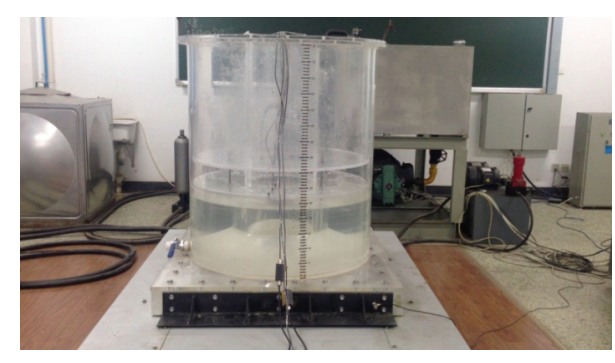

FIGURE 2: Experimental setup.

mounted on the supporting configuration by 4 connecting rods with knurled caps. The whole experimental tank was glued to a square shell which was screwed in 28 threaded holes on the shaking table.

The experimental setup included a computer, a laser displacement sensor, 3 pressure sensors, an acceleration sensor, a data acquisition tool, a set of commercial acquisition software COINV DASP, and some wires. The layouts and numbers of sensors are illustrated in Figure 3.

3.3. Experimental Procedure and Results. Tabulating in Table 2, a total of 6 experiment cases involving 4 different filling depths were carried out on the experimental setup with two configurations, first without internal components (a supporting configuration and an in-vessel roof) and then with internal components. For each case, sloshing experiments under multiple excitation amplitudes were carried out and several kinds of transient sloshing responses like sloshing wave height and slosh-induced impact pressure were recorded by data acquisition system. Measurements of the wave height are presented in Section 3.3.2, in cases without internal components. Impact pressure of sloshing is discussed in Section 3.3.3 in cases with internals.

3.3.1. Natural Frequency of Sloshing in First Mode. Liquid motion inside a tank in periodic movement is dominated by the liquid filling ratio, the amplitude and frequency of the tank motion, and the geometry of the tank. Considering the complex geometry of the experimental tank, the fundamental natural frequency of liquid was measured through sweep frequency response analysis (SFRA) experiment. The tank was partially filled with water, which was mixed with gouache paint and covered by pearl cotton on the surface. It was aimed to make the laser displacement sensor work normally by detecting object with surface that exhibited a diffuse reflection. From the research of Lu et al. [14], the influence of water level on natural frequency of sloshing water was quite small. Hence, the subtle variations of natural frequency with different filling depths can be ignored. The tank was filled up to a water level of $217 \mathrm{~mm}$, which was based on the length scale, for the measuring of liquid frequency. For the purpose of looking in detail at the resonance frequency of liquid, a logarithmic sweep with a range of $0.1 \sim 20 \mathrm{~Hz}$ was adopted. In addition, there are two types of control mode for driving the shaking table: Acceleration Control (AC) and Displacement Control (DC). At low frequency, about $0.1 \sim 4 \mathrm{~Hz}, \mathrm{AC}$ is used 
TABLE 2: Experiment sequences.

\begin{tabular}{lccc}
\hline Case number & Filling depth $H[\mathrm{~mm}]$ & $\begin{array}{c}\text { Distance between static water } \\
\text { surface and in-vessel roof } h[\mathrm{~mm}]\end{array}$ & Excitation amplitude $A[\mathrm{~mm}]$ \\
\hline $1^{*}$ & 217 & 43 & $12,24,36$ \\
2 & 177 & 83 & $12,24,36$ \\
3 & 197 & 63 & $12,24,36$ \\
4 & 237 & 23 & $12,24,36$ \\
\hline
\end{tabular}

$*: H$ and $h$ are based on the length scale.



Figure 3: Sensor layout.

and the displacement was controlled at the value of $2.0 \mathrm{~mm}$, since the displacement sensor achieved higher accuracy than acceleration sensor at low frequency range. However, DC is invoked in high frequency of $4 \sim 20 \mathrm{~Hz}$ and the acceleration was set with $0.650 \mathrm{~m} / \mathrm{s}^{2}$. The time history of sloshing displacement was transformed into frequency spectrum by a fast Fourier transform (FFT). By this analysis, the fundamental natural frequency of the liquid was evaluated to be $0.75 \mathrm{~Hz}$.

For an annular region formed by coaxial circular cylinder tank in periodic motion, the resonant frequencies of the liquid can be calculated from the linear potential theory as $[6,13]$

$$
\begin{aligned}
\omega_{m n}^{2}=\frac{g}{a} \xi_{m n} \tanh \left(\xi_{m n} \frac{H}{a}\right) \\
\quad m=0,1,2 \ldots, \infty, n=1,2,3, \ldots, \infty,
\end{aligned}
$$

where $g$ is the gravitational acceleration, $a$ is the outer radius of the tank, $\xi_{m n}$ are the positive roots related to the ratios of inner radius to outer radius of the tank, and $H$ is the still filling depth.

The calculating fundamental natural frequency from (6) is $0.887 \mathrm{~Hz}$, a little larger than the experimental result. It can be explained by the inclined bottom configuration of the tank.
3.3.2. Sloshing without the Roof. Due to the fact that the periods of sloshing water $(1 / f=1 / 0.75=1.33 \mathrm{~s})$ were far more longer than the site predominant period $(0.34 \mathrm{~s})$, the resonant three-cycle sine wave (RTCS) was assumed to be the input excitation according to the HAF0102 of nuclear safety guide in China, which is also a widely used evaluation method of seismic response in Japan. The load function can be written as $X=A \sin (2 \pi f t)$, where $X$ is the displacement of shaking table, $A$ is the amplitude of the sine wave, $f$ is the water natural frequency, and $t$ is the time which lasts for three periods of sine waves. In our experiments, the frequency of RTCS was set as $0.75 \mathrm{~Hz}$. When the frequency of excitation closed to the natural frequency, the liquid inside the experimental tank can exhibit strong oscillations, thus exerting a greater extent of sloshing and violent impact loads on the tank.

The experimental setup without internals was carried out for the experiment of seismic response about sloshing wave height. The sloshing wave height was measured through laser displacement sensor which was installed at point 1 near the outer tank wall which is showed in Figure 3. The acceleration transducer was installed at the bottom of the tank, in order to monitor the initial and final points of RTCS. Figure 4 reveals the vertical displacement of the wave in case 1 with $24 \mathrm{~mm}$ amplitude. It can be seen that the crest was almost 2 times 
TABLE 3: The maximum wave height.

\begin{tabular}{lcccc}
\hline Amplitude [mm] & Case 1 & Case 2 & Case 3 & Case 4 \\
\hline 12 & 79.191 & 65.272 & 67.103 & 98.9172 \\
24 & 227.355 & 163.953 & 190.414 & 264.142 \\
36 & 316.327 & 217.348 & 255.602 & 355.023 \\
\hline
\end{tabular}

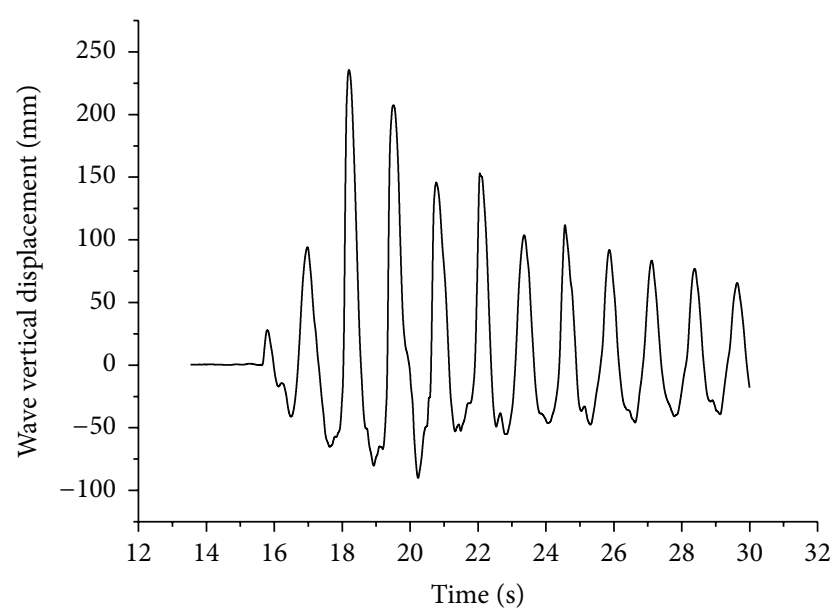

FIgURE 4: The vertical displacement time history of sloshing wave of case 1 with $24 \mathrm{~mm}$ amplitude.

size of the trough, which embodied an apparent feature of nonlinearity. Table 3 lists the maximum wave heights of case 1 to case 4 . Moreover, through the analysis of these data, for each case, the maximum wave heights increased as the excitation amplitudes grew and the increasing tendency gradually reduced, which was also a nonlinear behavior.

3.3.3. Sloshing with Roof. At the second part of the experiment, experiments with internals were carried out to measure the impact pressure of in-vessel roof. CYY8 dynamic pressure sensors with high frequency response were installed at measuring points 2, 3, and 4 on the roof, as showed in Figure 3, to present the radial pressure distribution.

Pressure variations were measured by dynamic piezoresistive pressure transducers in cases 1 to 4 . Figure 5 illustrates the impact pressures time history results in case 1 of measuring point 4 with $24 \mathrm{~mm}$ amplitude. According to the curve, the maximum impact pressure appeared when it came to the third wave of RTCS and then 2 small peaks came after it. These two small peaks can be explained by the inertia effect of water, which made water impact the inner roof despite the end of excitation. In addition, the gap between in-vessel roof and the tank wall would lead to a small portion of water splashing upward to the top surface of cap. Therefore, some negative values appeared intermittently in the measuring data showed in Figure 5.

As is shown in Figure 6, impact pressure distributions along the radial direction are presented in cases with different RTCSs and the same filling depth, $217 \mathrm{~mm}$. The impact pressure of point 2 was the minimum and the impact pressure of point 4 was the maximum, which showed that the impact pressure increases from inner wall to outer wall.



FIgURE 5: Time history of impact pressure about case 1 of point 4 with $24 \mathrm{~mm}$ amplitude.

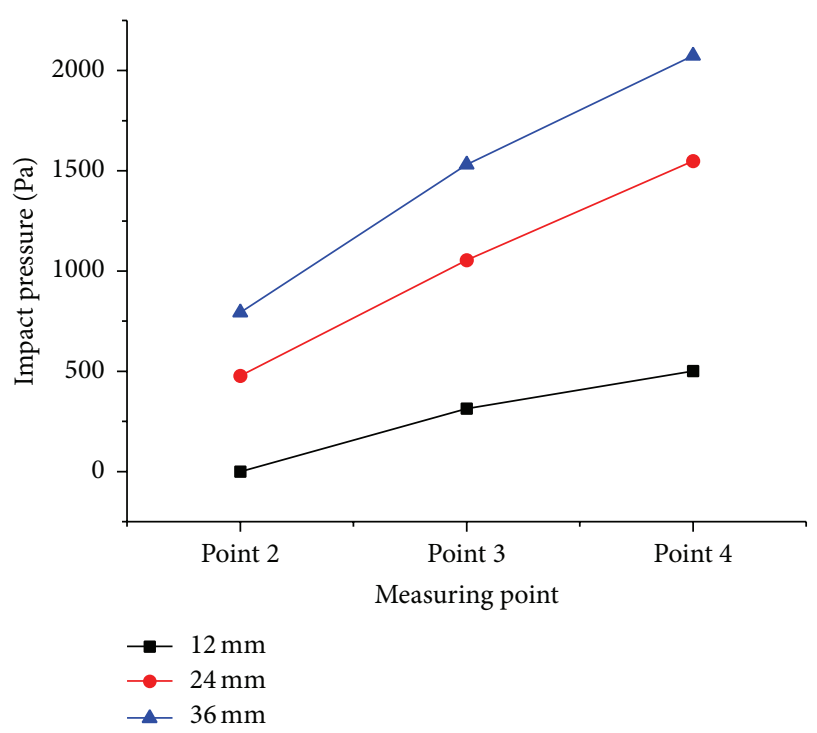

FIGURE 6: The distribution of impact pressure of case 1 with $24 \mathrm{~mm}$ amplitude.

During the operational process of experiments, there were some errors in experimental data owing to erroneous human input and the accuracy of measuring instruments to a certain extent. The use of laser displacement sensor, pressure sensors, and the indigenous vibration of shaking table caused significant errors in measurements. Besides, there also existed electromagnetic interferences during the operational process of shaking table, which adversely affected the accuracy of sensors especially pressure sensors, also contributed to the errors' list.

By computing the area integral of pressure, the experimental force data can be derived from the measuring pressure data with some conservative assumptions.

In our research, as illustrated in Figure 7, the area from inner wall to outer wall along $y$ axis is divided into 3 parts and the pressure of each part is assumed to be the measuring 


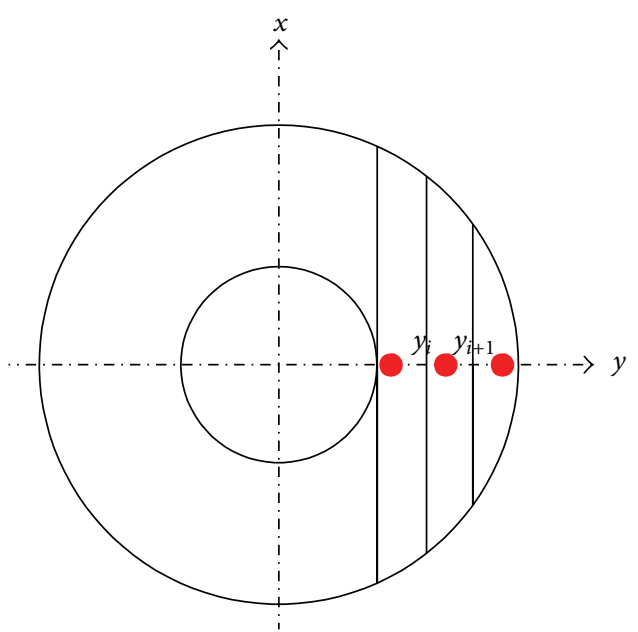

FIGURE 7: Schematic of area dividing.

value of the sensor which is installed at the corresponding part. Therefore, the impact force in each part can be written as in (7). The total impact force is the sum of that in each part which is showed in (8). Consider the following:

$$
\begin{aligned}
& F_{i}=\int_{-\sqrt{R^{2}-y^{2}}}^{\sqrt{R^{2}-y^{2}}} d x \int_{y_{i}}^{y_{i+1}} p_{i} d y, \\
& F_{t}=\sum_{i=1}^{n} F_{i} \quad i=1,2,3,
\end{aligned}
$$

where $F_{i}$ and $p_{i}$ are the impact force and impact pressure in each part, $R$ is the outer radius of cylinder tank, and $F_{t}$ is the total impact force.

In our cases, the total impact forces of different conditions are listed in Table 4.

\section{Analysis of Experimental Results}

In Section 2, we proposed a simplified method to estimate the impact pressure. So we list the results of simplified method and measured data. We used the calculated impact pressure (CIP) indirectly to represent the results of simplified method. The measured data were called the measured impact pressure (MIP) directly. The comparison between calculated pressure and measured pressure is listed in Table 5 .

According to Table 5, CIP are lower than MIP under small amplitudes of RTCS. A possible reason is the influence of air motion. In our experiment, the water tank cannot be thoroughly sealed. Therefore, air in the ring cavity can move with the vigorous sloshing of water. Air motion is the primary influential factor under low level of excitation. However, it is considered that small excitation has really subtle effect in engineering practice. As for the higher excitation level, CIP are larger than MIP under medium and high level of excitations. With a certain error of no more than $35 \%$, this method of impact pressure calculation is considered to be overpredictive. Therefore, similar to the results of Lu's [15] research, it
TABLE 4: Total impact force from measuring data.

\begin{tabular}{lccc}
\hline Case no. Filling depth [mm] & Amplitude [mm] & Impact force [N] \\
\hline \multirow{3}{*}{$217 \mathrm{~mm}$} & 12 & 11.31001 \\
& & 24 & 60.37129 \\
& 36 & 91.28681 \\
\hline \multirow{3}{*}{2} & $177 \mathrm{~mm}$ & 24 & 4.370317 \\
& & 36 & 25.62775 \\
& \multirow{3}{*}{$197 \mathrm{~mm}$} & 12 & 41.8155 \\
\hline & & 24 & 7.423492 \\
& & 36 & 36.49382 \\
& & 12 & 56.19478 \\
\hline \multirow{3}{*}{4} & $237 \mathrm{~mm}$ & 24 & 84.23761 \\
& & 36 & 117.9033 \\
\hline
\end{tabular}

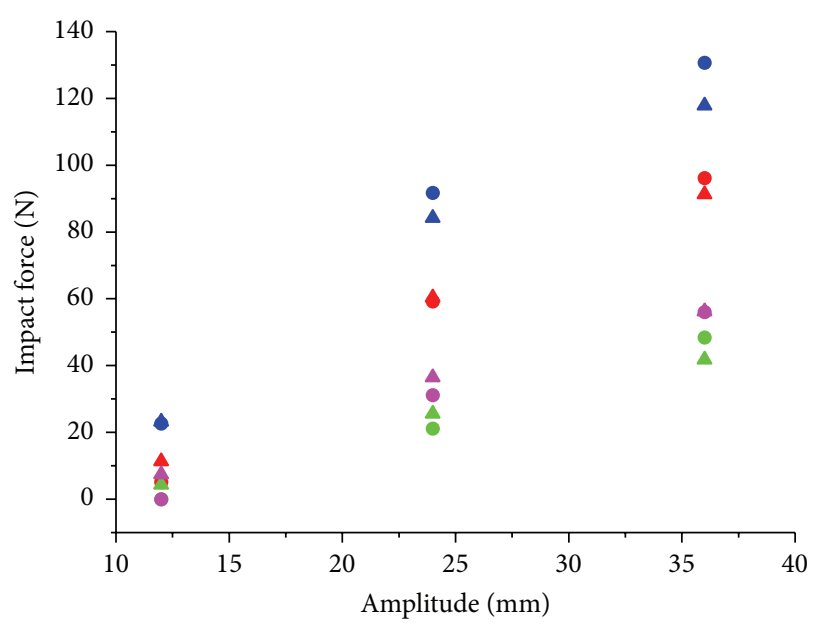

- Case 1 by experimental force

- Case 1 by calculated force

$\triangle$ Case 2 by experimental force

- Case 2 by calculated force

- Case 3 by experimental force

- Case 3 by calculated force

- Case 4 by experimental force

- Case 4 by calculated force

FIGURE 8: Comparison of calculated force and experimental force.

can be concluded that the calculation method is reasonable and relatively conservative.

According to the simplified calculation equation for impact force proposed in Section 2, we obtained the impact force of simplified method which was called calculated force here. Showed in Section 3.3.3, the experimental force data was called experimental force here. Figure 8 compares the calculated force and experimental force. The calculated force is lower than, though generally coinciding with, the experimental force in smaller wave height cases. However, calculated force becomes close to the experimental force and then exceeds it due to the increase of maximum sloshing wave height.

By the comparison of calculated results and experimental results, some future works are left. First, there should be some holes on the in-vessel roof to reduce the influence of air motion during experimental procedure. Second, longer 
TABLE 5: The comparison between calculated pressure and measured pressure.

\begin{tabular}{|c|c|c|c|c|}
\hline Case no. & Amplitude [mm] & Measured pressure (MIP) $[\mathrm{Pa}]$ & Calculated pressure (CIP) $[\mathrm{Pa}]$ & Error $(\%)$ \\
\hline \multirow{3}{*}{1} & 12 & 500.62 & 354.6718 & -29.1536 \\
\hline & 24 & 1549.30 & 1806.679 & 16.6126 \\
\hline & 36 & 2074.05 & 2678.605 & 29.14851 \\
\hline \multirow{3}{*}{2} & 12 & 263.27 & 0 & -100 \\
\hline & 24 & 913.30 & 793.3394 & -13.1349 \\
\hline & 36 & 1243.44 & 1316.61 & 5.884514 \\
\hline \multirow{3}{*}{3} & 12 & 397.514 & 40.209 & -89.8856 \\
\hline & 24 & 1221.34 & 1248.657 & 2.236658 \\
\hline & 36 & 1579.48 & 1887.5 & 19.50133 \\
\hline \multirow{3}{*}{4} & 12 & 757.21 & 743.9886 & -1.74607 \\
\hline & 24 & 1753.86 & 2363.192 & 34.74232 \\
\hline & 36 & 2445.99 & 3253.825 & 33.02691 \\
\hline
\end{tabular}

duration time experiments with more pressure sensors and displacement sensors are needed to investigate the impact pressure distribution and computation of impact force.

\section{Conclusions}

(1) In order to estimate the impact pressure imposed on the roof of water storage tank of AP1000, we proposed a simplified method, which regarded approximately the difference between maximum wave height and roof height as the impact pressure head.

(2) In order to verify the above method, we built a scaled experimental facility.

(3) Using the experimental facility, we measured the maximum sloshing wave height in the case of not having roof and the impact pressure in the case of having roof.

(4) By the above experiment, the simplified method was considered to be conservative with a certain error of no more than $35 \%$.

\section{Competing Interests}

The authors declare that they have no competing interests.

\section{Acknowledgments}

The project was sponsored by National Science and Technology Major Project of the Ministry of Science and Technology of China (2015ZX06004002-003).

\section{References}

[1] R. A. Ibrahim, Liquid Sloshing Dynamics: Theory and Applications, Cambridge University Press, New York, NY, USA, 2005.

[2] N. C. Pal, P. K. Sinha, and S. K. Bhattacharyya, "Finite element coupled slosh analysis of rectangular liquid filled laminated composite tanks," Journal of Reinforced Plastics and Composites, vol. 18, no. 15, pp. 1375-1407, 1999.
[3] Y.-S. Choun and C.-B. Yun, "Sloshing analysis of rectangular tanks with a submerged structure by using small-amplitude water wave theory," Earthquake Engineering and Structural Dynamics, vol. 28, no. 7, pp. 763-783, 1999.

[4] J. G. Li, Y. Hamamoto, Y. Liu, and X. Zhang, "Sloshing impact simulation with material point method and its experimental validations," Computers \& Fluids, vol. 103, pp. 86-99, 2014.

[5] M. Eswaran, U. K. Saha, and D. Maity, "Effect of baffles on a partially filled cubic tank: numerical simulation and experimental validation," Computers \& Structures, vol. 87, no. 3-4, pp. 198-205, 2009.

[6] K. Fujita, T. Ito, and K. Okada, "Seismic response of liquid sloshing in the annular region formed by coaxial circular cylinders," Engineering Computations, vol. 2, no. 4, pp. 299-306, 1985.

[7] J. C. Virella, C. A. Prato, and L. A. Godoy, "Linear and nonlinear 2D finite element analysis of sloshing modes and pressures in rectangular tanks subject to horizontal harmonic motions," Journal of Sound and Vibration, vol. 312, no. 3, pp. 442-460, 2008.

[8] S. K. Nayak and K. C. Biswal, "Nonlinear seismic response of a partially-filled rectangular liquid tank with a submerged block," Journal of Sound and Vibration, vol. 368, pp. 148-173, 2016.

[9] H. Akyildiz and E. Ünal, "Experimental investigation of pressure distribution on a rectangular tank due to the liquid sloshing," Ocean Engineering, vol. 32, no. 11-12, pp. 1503-1516, 2005.

[10] Y. K. Song, K.-A. Chang, Y. Ryu, and S. H. Kwon, "Experimental study on flow kinematics and impact pressure in liquid sloshing," Experiments in Fluids, vol. 54, no. 9, article 1592, 2013.

[11] A. Souto-Iglesias, G. Bulian, and E. Botia-Vera, "A set of canonical problems in sloshing. Part 2: influence of tank width on impact pressure statistics in regular forced angular motion," Ocean Engineering, vol. 105, pp. 136-159, 2015.

[12] F. Pistani and K. Thiagarajan, "Experimental measurements and data analysis of the impact pressures in a sloshing experiment," Ocean Engineering, vol. 52, pp. 60-74, 2012.

[13] H. N. Abramson, "The dynamic behavior of liquids in moving containers, with applications to space vehicle technology," Tech. Rep. NASA-SP-106, NASA, Washington, DC, USA, 1966. 
[14] D. Lu, Y. Liu, and X. Zeng, "Experimental and numerical study of dynamic response of elevated water tank of AP1000 PCCWST considering FSI effect," Annals of Nuclear Energy, vol. 81, pp. 7383, 2015.

[15] D. Lu, "Sloshing response of the free surface in the main vessel of CEFR excited by 3 sine waves," Chinese Journal of Nuclear Science and Engineering, vol. 23, pp. 306-310, 2003. 


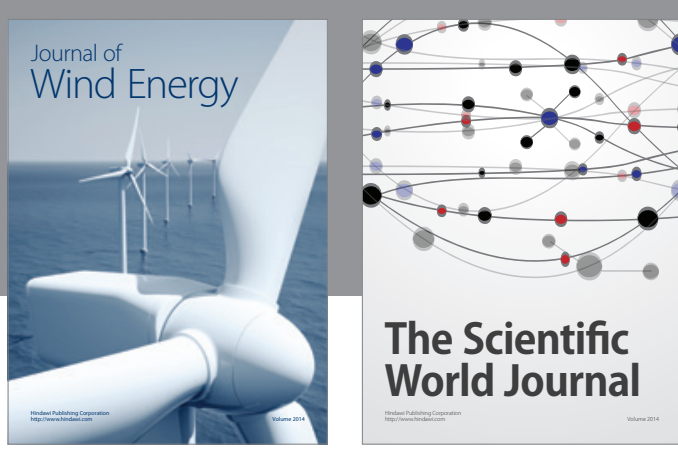

The Scientific World Journal
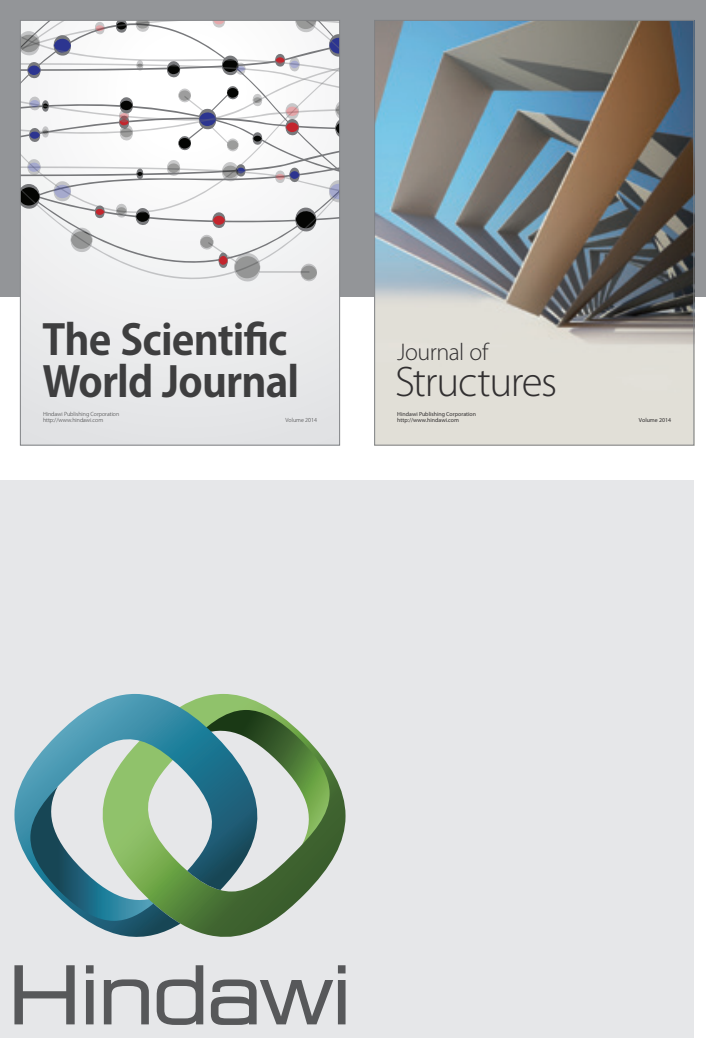

Submit your manuscripts at

http://www.hindawi.com
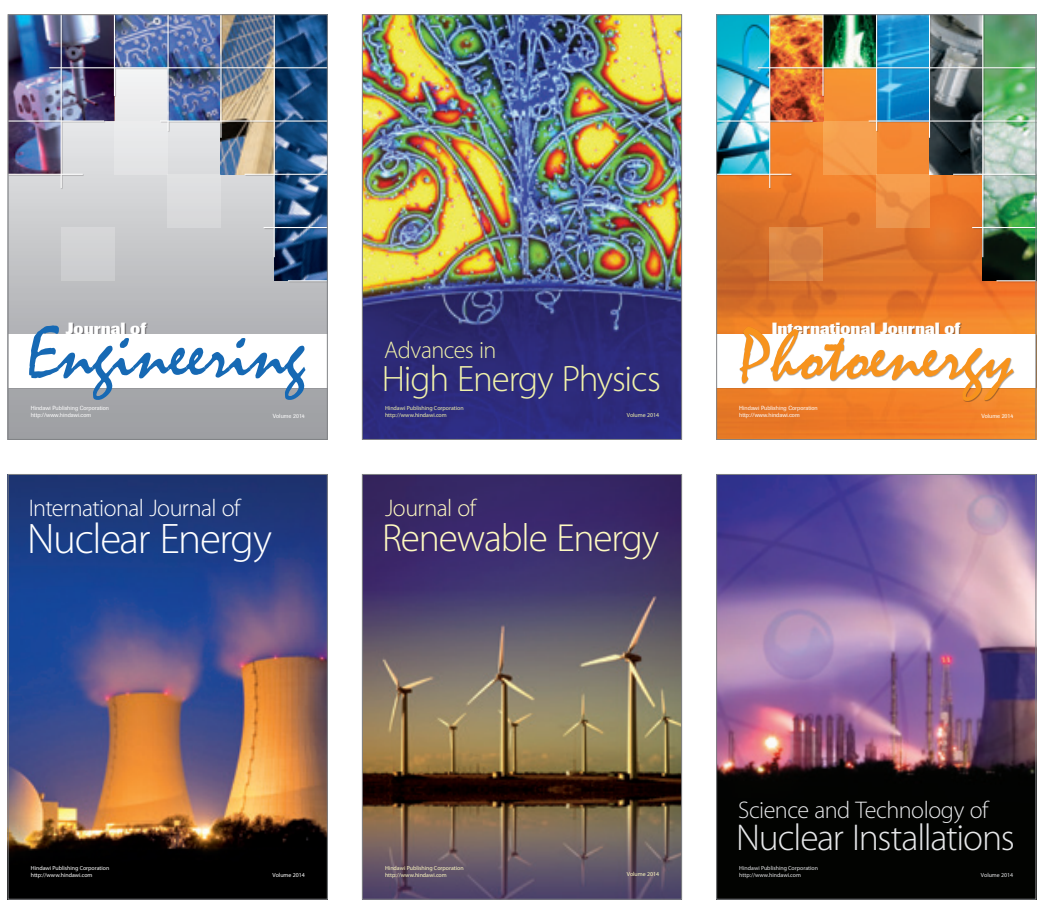
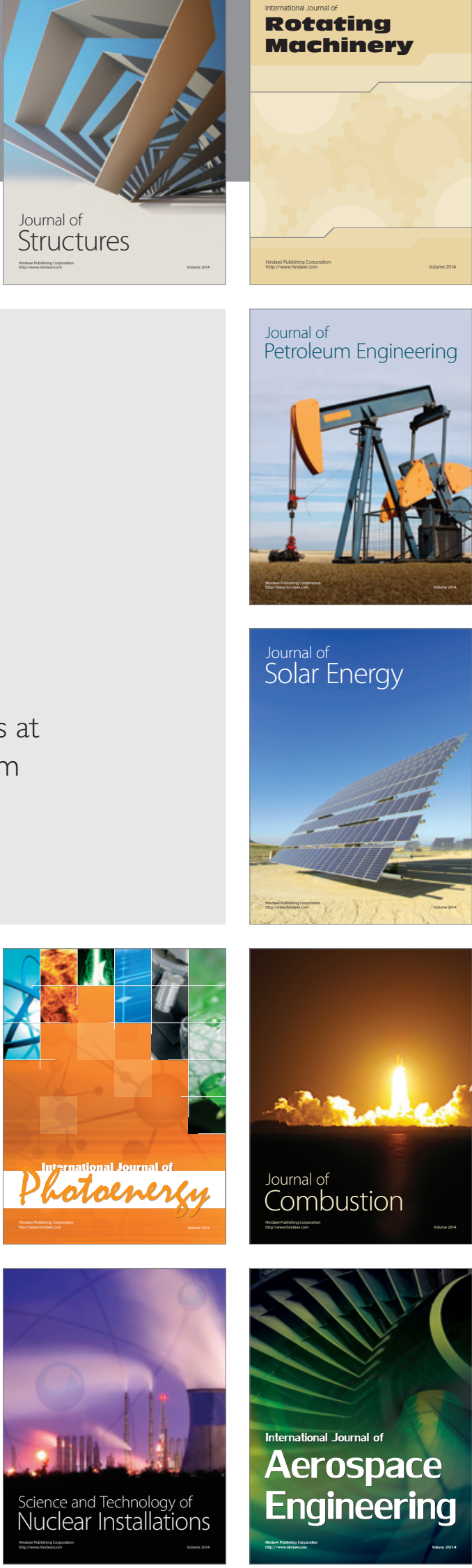